\title{
A One-Stop BSS Analysis Method of Integrated System Signals Based on Wavelet Transform, FastICA and SOM Hongyi $\mathrm{Li}^{1,2}$, Liantao $\mathrm{Ma}^{1,2}$, Bin Chen ${ }^{2}$ and Di Zhao ${ }^{1, *}$
}

1. LMIB, School of Mathematics and Systems Science, Beihang University, Beijing 100191, China

2. School of Software Engineering, Beihang University, Beijing 100191, China

*. Corresponding author (E-mail: zdhyl2010@163.com)

Keywords: blind source separation; wavelet transform; FastICA; SOM

\begin{abstract}
This paper proposes a new one-stop blind source separation analysis method by combining wavelet transform, FastICA and self-organization mapping network. Firstly, we suppress the Gauss noises by using wavelet transform. Secondly, we separate the mixed signals utilizing FastICA to get the independent components. Finally, we cluster the signals with SOM to reveal the latent relationship of independent signals. Experimental results have shown the validity and effectiveness of the proposed method.
\end{abstract}

\section{Introduction}

Most of the measurement/detection method and equipment of a large-scale integrated system work directly on the whole system. It is generally difficult to exactly acquire signals emitted by all the electronic components. As a result, a one-stop method is desirable to suppress disturbing elements, and extract useful information on the electronic component from signals of the whole system, and finally could provide accurate math simulation model for further system design, analysis, prediction and evaluation.

Aiming at the signals of integrated system, this paper uses the ICA to pretreat the mixed data which is collected. Separate the mixed signals with no prior information to get the independent signals produced by different parts of system.

To solve the BSS problem, there are some methods now, such as K-means clustering [1], EASI [2], and ICA [3]. ICA is the main solution of BSS. And FastICA [4] is being widely used, because of its good flexibility, robustness and fast convergence.

The performance of FastICA is sensitive to the initial value of the separating matrix input weight. Now, researchers proposed many improvements about FastICA. Paper [5] suggests to improve the FastICA in this aspect. First, compare the performance with the best functional simulation selected from three different nonlinear functions, and adjust the parameter of best function to make further improvement. Then, compute the initial value by the best simulating function. The experiment result shows that, this method can solve the problem that the performance is sensitive to the initial value. Besides, it can avoid the nonuniform convergence and improve the result of separation.

Paper [6] suggests that, when the FastICA is used in dealing with big data (such as image processing), the fifth-order convergence Newton iteration method can be used to accelerate the convergence speed. The simulation result shows that the improved FastICA has better separating feature and less convergence counts.

Most of the improvement methods now are aiming at the separation method itself, trying to improve the separating result and the convergence speed. There are little researches importing the intelligence artificial algorithms such as artificial neural network, to build the one-stop processing method for integrated system signals including noise.

The conventional ICA method is sensitive to noise. It cannot separate valuable signals correctly when there is serious noise pollution. To solve this problem, this paper proposed the improved method combing the wavelet transform and FastICA. Reduce the noise of collected signals with wavelet threshold method. Improve the signal to noise ratio, then separate the signals using FastICA. 
When using FastICA, the sequence of output vectors is uncertain. It is hard to confirm which source the extracted signal corresponds to [7]. And the physical meaning of independent components cannot be identified easily. The result of ICA is hard to utilize directly. The automatic identification system is desirable [8]. So we take the features of independent components separated as input vectors, and do the cluster analysis by SOM. The result can lays foundation for further analyzing the signals.

The rest of this paper is organized as follow: Section 2, 3, 4 introduce the basic concept of wavelet transform, FastICA and SOM. Section 5 explains our one-stop BSS analysis method. In section 6, we have completed a simulation experiment to verify the effectiveness of our method.

\section{Wavelet Transform}

Wavelet transform is considered to provide a suitable analysis structure to investigate irregular and transient phenomena in signals [9], which is widely used in denoising [10][11]. This technique focuses on the details of the signals, and analyzes the partial information of low frequency and high frequency in scale spaces. By thresholding the components separated, the reconstructed signal has a low level of noise, while maintaining the details of signal.

The wavelet threshold de-noising algorithm is describes in Table 1 [9]:

TABLE I. PROCEDURE OF THE WAVELET THRESHOLD DE-NOISING ALGORITHM

Input: Observed Signal

Output: Signal with low noise

Step1. Divide input signals into several stationary segments and estimate the surd and sonant character for each segment.

Step2. Calculate the 1 to 5 order high frequency coefficients for each segment

Step3. Estimate threshold for high frequency coefficients in each order

Step4. Reconstruct the signals by 1 to 5 order high frequency coefficient from wavelet domain.

\section{FastICA}

FastICA (Fast Independent Component Analysis) is a popular algorithm for blind source separation (BSS) of independent sources from their linear mixtures. It attempts to find a set of independent components by estimating the maximum negative entropy [12]. The algorithm iteratively searches for the weight set $w_{i}$ of a neural network from a data set $\mathrm{X}$ via the procedure below [13]:

TABLE II. PROCEDURE OF FASTICA

Input: Observed Signals

Output: Independent Components

Step1. Initialize weighting vector. Set the initial value of learning rate

Step2. Compute the Euclidean Distance between training samples and weighting vector for every output node $D\left(w_{j}, x_{n}\right)=\sqrt{\sum\left(w_{i j}-x_{n i}\right)^{2}}$, and find the winning node $\mathrm{J}$ whose value of $\mathrm{D}$ is the minimum.

Step3. Set $w \leftarrow E\left\{z g\left(w^{T} z\right)\right\}-E\left\{g\left(w^{T} z\right)\right\} w$, where $g$ is a contrast function defined as $g_{1}(y)=\tanh \left(a_{1} y\right)$ (the constant $a_{1}$ is usually in range $[0,1)$, and $g_{2}(y)=y \exp \left(-\frac{y^{2}}{2}\right)$

Step4. Normalize w. i.e. let $w \leftarrow w /\|w\|$

Step5. If not converged, go back to Step 3

\section{Self-Organization Mapping Network}

SOM is proposed by the expert of artificial neural network, professor Kohonen, in 1981 [14]. It is widely used in data classification, pattern identification, image processing and other fields [15]. 
This network adopts unsupervised learning rule. The basic procedure of SOM algorithm is [2]:

TABLE III. PROCEDURE OF SOM

Input: characteristic values of independent components

Output: results of clustering

Step1. Initialize weighting vectors. Set the initial value of learning rate.

Step2. Compute the Euclidean Distance between training samples and weighting vector for every output node: $D\left(w_{j}, x_{n}\right)=\sqrt{\sum\left(w_{i j}-x_{n i}\right)^{2}}$, and find the winning node $\mathrm{J}$ whose value of $\mathrm{D}$ is the minimum.

Step3. Adjust the weighting vector. Because of the lateral inhibition between competitive layer neurons, just adjust the weight in the field. The adjustment function is:

$$
w_{i j}(t+1)=w_{i j}(t)+\eta\left(x_{n i}-w_{i j}(t)\right) .
$$

Step4. Adjust learning rate and the radius of field, till characteristic patterns stop changing or achieve the frequency of training. Save the weighting vector trained. Otherwise, go back to Step2.

\section{One-Stop Analysis Method}

To analyze and utilize the valued information in observed mixed signals conveniently, we proposed the one-stop analysis method.

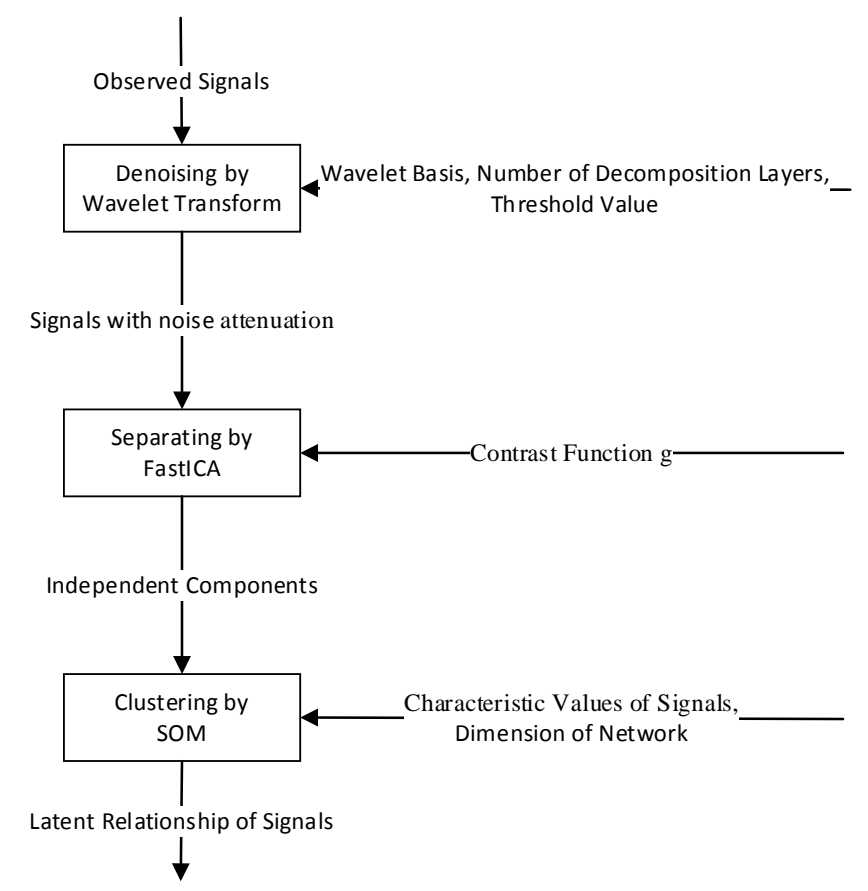

Figure 1. $\quad$ Procedure of One-Stop BSS Analysis Method

table iv. Procedure of ONE-Stop BSS AnAlysis Method

Input: observed signals

Output: independent components and their latent relationship

Step1. Select appropriate wavelet basis, and determine the number of its decomposition layers. Do the wavelet decomposition with signals which contains noise.

Step2. Select a threshold value for wavelet high frequency coefficient, and denoise the wavelet coefficient.

Step3. Wavelet reconstitution. Get the denoised source signals after the wavelet transform and reconstitution of denoised wavelet coefficient.

Step4. Whiten the mixed signals

Step5. Initial vector w $(0)$

Step6. Select appropriate function g. Do the iterative computation $w \leftarrow E\left\{z g\left(w^{T} z\right)\right\}-E\left\{g\left(w^{T} z\right)\right\} w$. 
Step7. Normalize vector $\mathrm{w}$

Step8. Stop the iteration, and output the vector $w$, if it is convergent. If it is not convergent or achieve the max iterations, turn to step 6.

Step9. Select characteristic values according to the feature of signal, and calculate the characteristic values of the independent signals.

Step10. Import the matrix of characteristic values into SOM network

Step11. Set the dimension of SOM network, according to the numbers of signals. Experiment on different iterations.

Step12 Cluster the independent signals into different groups.

If it cannot identify the different signals clearly, turn to step 4 and select other characteristic values.

If the clustering result is too close/dispersive, turn to step 6 , set bigger/smaller dimension.

\section{Experiment and Analysis}

To test and verify the performance the one-stop method proposed above, we select the four simulation signals below and do the experiment: Doppler signal, sinusoidal signal $\sin (2 \pi \times r)$, cosine signal $\cos (2 \pi \times 2 r)$, quadchirp signal [16] as shown in Figure 1 .

In our experiment, we set the sampling frequency as $100 \mathrm{~Hz}$. There are 1024 points between 1 and 10 . The hybrid matrix $A$ is randomly generated. The white Gaussian noises are added to every observed signal. Then we get the simulating observed signals as shown in Figure 2.

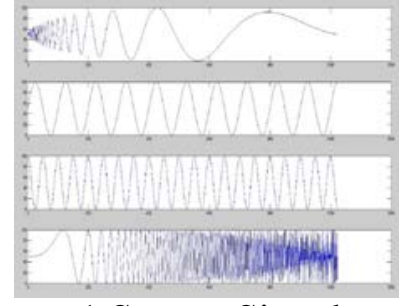

Figure 1 Source Signals

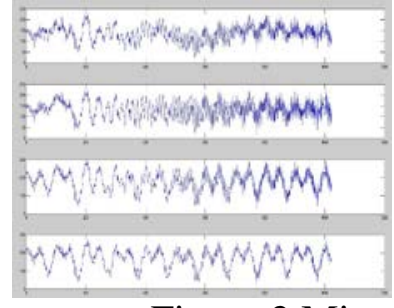

Figure 2 Mixed Signals

We select the db1 wavelet to do the 2 layer decomposition, and select g1 nonlinear function in FastICA.

Source signals are mixed randomly and polluted by noises. We can get 4 independent component by using FastICA to separate signals directly. The separating results are shown in Fig. 5, from which we can see that there are lots of noise mixed in independent components. This suggests that FastICA has a limited inhibiting capacity to white Gaussian noise, and is sensitive to noises. Therefore, the features of desired signals cannot be identified clearly.

Figure 4 shows the separating results by using FastICA after being preprocessed through the wavelet transform, from which we can see that the signal to noise ratio is improved obviously. The details of independent components got from denoised-FastICA are clearer than Figure 5.

To quantitatively evaluate the performance of the separating results, we calculate the correlation between the separated singles in Fig. 4, and the original signal shown in Figure 1. According to Table 5, the correlation coefficients between source signals and separated signals using the proposed method are pretty high, which could show the validity of the proposed method.

TABLE V. PROCEDURE OF SOM

\begin{tabular}{|c|c|c|c|c|}
\hline Source Signals & S1 & S2 & S3 & S4 \\
\hline $\begin{array}{c}\text { Separated Signals (without } \\
\text { denoising) }\end{array}$ & Y1 & Y2 & Y4 & Y3 \\
\hline $\begin{array}{c}\text { Correlation Coefficients (without } \\
\text { denoising) }\end{array}$ & 0.6279 & 0.8117 & 0.4930 & 0.3915 \\
\hline $\begin{array}{c}\text { Separated Signals } \\
\text { (with denosing) }\end{array}$ & Y2 & Y3 & Y1 & Y4 \\
\hline $\begin{array}{c}\text { Correlation Coefficients } \\
\text { (with denosing) }\end{array}$ & 0.9875 & 0.9840 & 0.9913 & 0.9684 \\
\hline
\end{tabular}



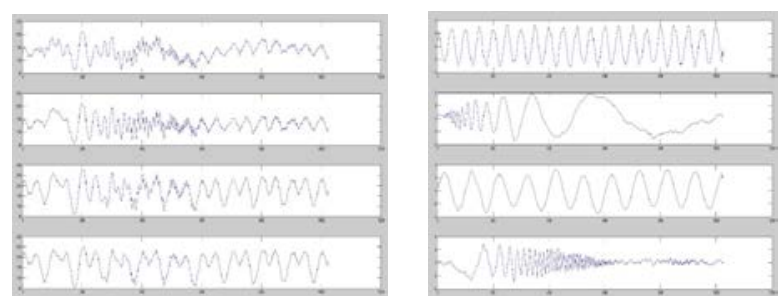

Figure 3 Signals with Denoising Figure 4 Signals Separated by FastICA

In our experiment setting of clustering, we set the dimension of SOM network as $2 * 2$, and select skewness, range, coefficient of kurtosis and numbers of wave peaks as characteristic values.

table VI. Clustering Results

\begin{tabular}{|c|c|c|c|c|}
\hline Independent Signals & 1 & 2 & 3 & 4 \\
\hline $\begin{array}{c}\text { Group 1 } \\
\text { (sin and cos signals) }\end{array}$ & 1 & & & \\
\hline $\begin{array}{c}\text { Group 2 } \\
\text { (Doppler signal) }\end{array} \begin{array}{c}\text { Group 3 } \\
\text { (Quadchirp Signal) }\end{array}$ & & 1 & 1 & 1 \\
\hline
\end{tabular}
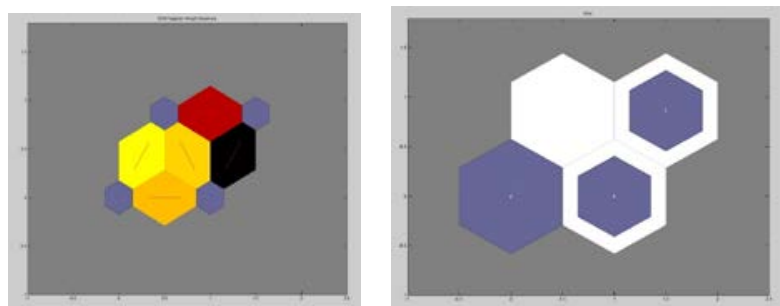

Figure 6 SOM Neighbor Weight Distances

Figure 7 Hits

According to Table 6, SOM network cluster the signals into 3 groups with the characteristic values, corresponding to the Doppler signal, sinusoidal signal and quadchirp signal. The result of cluster is perfect. In practical applications, we can increase the numbers of signals to improve the clustering performance.

\section{Conclusions}

In this paper, we propose a novel one-stop BSS Analysis Method of Integrated System Signals Based on Wavelet, FastICA and SOM. The method can separate the mixed observed signals and cluster the independent components to reveal the latent relationship of signals.

FastICA is an effective separating algorithm. But, due to its noise sensitiveness, there are still lots of noise mixed in separated results. Combing with the Denoising ability of wavelet transform, we can improve the separating results greatly. Moreover, FastICA cannot determine the sequence of independent components. While SOM network has the ability to do the data mining with signals, covering the shortage of FastICA.

The experiment shows that the one-stop BSS analysis method is suitable to deal with signals produced by integrated systems. The result lays the foundation for further analyzing signals emitted by different parts in system.

\section{Acknowledgment}

The research work was supported by National Natural Science Foundation of China under Grant No. 61379001 and Key Project of Beihang University under Grant No. 201412 ("Exploration and Practice of Innovation Ability Cultivation in Mathematical Education”). 


\section{References}

[1] Q. M. Yi, "Blind source separation by weighted K-means clustering,” Journal of Systems Engineering and Electronics, vol.19, no.5, pp.882-887, 2008.

[2] M. L. Zhang, "Survey on SOM Algorithm, LVQ Algorithm and their variants," Computer Science, vol.29, no.7, 2002.

[3] H. Y. Li, M. Ye and D. Zhao, "An improved ICA algorithm based on the negative entropy and simulated annealing algorithm,” Applied Mechanics and Materials, vol. 411-414, pp. 11251128, 2013.

[4] D. Zhao, H. Lu and H. Y. Li, “An ICA and AIS Based Method for Electromagnetic Compatibility Analysis,” Advanced Materials Research, vol. 446-447, pp. 547-550, 2013.

[5] Z. M. Li, and G.K. Yang. "Blind Separation of Mixed Audio Signals Based on Improved FastICA,” in 6th International Congress on Image and Signal Processing (CISP 2013), 2013.

[6] F. Zhao, et al, "An Improved Method for the FastICA Algorithm," in International Joint Conference on Neural Networks(IJCNN), pp.2925 - 2929, 2011.

[7] A. Hyvärinen, J. Karhunen, E. Oja, Independent component analysis, John Wiley \& Sons, U.S.A, 2004.

[8] H.Y. Li, Y. Fu and D. Zhao, "Identification of Power Quality Disturbances Based on FFT and Attribute Weighted Artificial Immune Evolutionary Classifier,” Applied Mechanics and Materials, vol.530-531, pp.277-280, 2014.

[9] H. Li, et al. "Blind separation of noisy mixed speech signals based on wavelet transform and Independent Component Analysis," in ICSP, 2006.

[10] M. Alfaouri, K. Daqrouq. "ECG signal denoising by wavelet transform thresholding," American Journal of applied sciences, vol.5, no.3, pp.276, 2008.

[11] S. A. Ouadfeul, L. Aliouane, "Random seismic noise attenuation data using the discrete and the continuous wavelet transforms,” Arabian Journal of Geosciences, pp.1-7, 2013.

[12] X. L. Li, and T. Adali, "Independent component analysis by entropy bound minimization," IEEE Transactions on Signal Processing, vol.58, no.10, pp.5151-5164, 2010.

[13] P. Tichavský, Z. Koldovský, and E. Oja, "Performance analysis of the FastICA algorithm and Cramér-Rao bounds for linear independent component analysis,” IEEE Trans. Signal Process, vol.54, no.4, pp.1189-1203, 2006.

[14] T. Kohonen, “The self-organizing map,” Proceedings of the IEEE, vol.78, no.9, pp.1464-1480, 1990.

[15] G. J. Meschino, D. S. Comas, V. L. Ballarin, et al., "Automatic design of interpretable fuzzy predicate systems for clustering using self-organizing maps,” Neurocomputing, vol.147, pp. 47-59, 2015.

[16] J. Kunisch, J. Pamp, "Wideband car-to-car radio channel measurements and model at 5.9 GHz,” Vehicular Technology Conference, pp.1-5, 2008. 